\title{
Role Assignment Adaptation: An Intentional Forgetting Approach
}

\author{
Ingo J. Timm, Lukas Reuter, and Jan Ole Berndt \\ Center for Informatics Research and Technology \\ Trier University \\ $\{$ itimm, reuter, berndt $\} @$ uni-trier.de
}

\begin{abstract}
In organizations the distribution of tasks is a rising challenge in complex and dynamic environments. By structuring responsibilities and expectations for task processing in roles, organizations provide a transparent approach for collaboration. However, if tasks are being generated unexpectedly, actors who enact multiple roles might be overloaded in dynamic environments. By focusing on relevant information in terms of an intentional forgetting mechanism, actors could overcome these overload situations. Therefore, we provide an agent-based simulation to model and analyze effects of intentional forgetting by adapting role assignments in dynamic environments. The agent architecture utilizes separated revision functions to control an agent's perception and belief acquisition to focus on relevant information. The model is tested using a case-study in a simulated emergency response scenario. The simulation results show that adapting role assignments at runtime improves team performance significantly.
\end{abstract}

\section{Introduction}

Modern organizations are driven by current trends such as the digital transformation or cyber-physical-systems as in Industry 4.0. In particular, these organizational developments lead to more diverse and complex tasks which cannot be handled by single actors anymore. Hence, teamwork is an important component of modern work environments in which tasks are divided into subtasks and distributed across the team. Due to the rising responsibilities of technical systems in modern organizations, the combination of human and technical workforce is becoming more important. Thus, cyber-physical-social-systems extend the classical approach by a human component which interacts with machines [1,2]. In Industry 4.0, physical machines are complemented by a virtual representative to communicate and to manage distributed processes in the team. In order to allow for socio-digital communication, human worker need also to be represented virtually. From a distributed artificial intelligence (DAI) perspective, these representatives are modeled as intelligent agents. In DAI, multiagent systems (MAS) are used to couple agents and distribute tasks across a team [3]. Furthermore, both kind of actors have to be represented in an organization formally to plan their activities within an integrated approach. Typically, roles are used as structural elements which couple expectations and responsibilities for its incumbents [4]. Actors in organizations may enact multiple roles which results in more responsibilities for different tasks.

In dynamic environments, tasks may occur unforeseen which leads to a high workload for actors with multiple roles due to their responsibilities. When humans deal with high workload situations, context switches between tasks lead to stress and reduced efficiency. Hence, they may focus on particular tasks individually to cope with overload situations [5]. Focusing on single tasks allows for a more efficient task processing due to less task switching costs. But it lowers the robustness of a team in case of disturbances [6]. That is, if team member are unavailable, urgent tasks may not be processed since the other member are specialized on different areas of expertise. In psychological research, focusing on relevant information at task processing can be defined as an intentional forgetting (IF) process $[7,8]$. When irrelevant information is ignored, humans tend to have more capacity for the task at hand. In order to focus on relevant information and prevent information overload, role assignment adaptation seems as an appropriate mechanisms to manipulate the behavior of organization members. That is, if one enacts fewer roles, their task responsibilities decreases and their gained cognitive capacity should result in a better work performance from a team perspective.

However, overload situations do not only effect humans but also technical systems $[9,10]$. In 
a socio-digital work environment the capabilities and workload of technical systems have to be taken into account to allow for an integrated role configuration approach of the whole organization. Due to rising responsibilities for tasks based on role configurations, switching between them is causing delays and communication overhead for technical systems. Therefore, we transfer the concept of intentional forgetting as a specialization approach to socio-digital environments by means of agent-based modeling. First simulation experiments show that configuring teams in a specialist role assignment configuration outperform generalist configurations in terms of effectivity [11]. Hence, adapting role assignments during runtime should increase team performance.

This paper contributes by modeling and simulating role assignment adaptation at runtime to analyze effects of intentional forgetting. When actors perceive a heavy workload, role assignments are adapted by specializing on certain tasks to act more efficiently. These role assignment adaptations force agents to focus on specific tasks which reduces context switches and allows to intentionally forget irrelevant information. We use a Belief-Desire-Intention (BDI) agent architecture to model and implement IF. More specific, this model contributes role-dependent functions for revising agent beliefs which allows for a modularized implementation to focus on certain roles. These functions are modified using a meta-reasoning component to adapt an agent's internal reasoning process. The model is evaluated using a serious game environment for team collaboration and task distribution which simulates an emergency response scenario. This multiagent-based simulation is a first step of evaluating workforce performance in socio-digital, dynamic and flexible work environments and is a foundation for optimizing role configurations in specific teamwork scenarios.

The remainder of this paper is structured as follows. Section 2 provides an overview of teamwork processes from a psychological perspective and introduces intentional forgetting as specialization in teamwork processes. In Section 3, foundations for agent collaboration in organizations and reorganization are discussed. Moreover, Section 4 presents the adaptation approach using intentional forgetting in agent organizations to adapt role assignment based on specialization principles. Section 5 presents a serious game environment and provides a case-study in an emergency response scenario to test the effects of reorganizing role assignments for evaluation. In Section 5.2 results from simulation experiments are shown and discussed. Section 6 concludes this article.

\section{Specialization in teamwork: an intentional forgetting approach}

In psychological group-research, teams can be defined as collective information-processing systems [12]. Team members memorize knowledge required for their tasks, they specialize on particular areas of expertise, or they share knowledge and information with each other [13, 14]. When working together, it is important for team members to share their knowledge about task and team relevant information with each other to facilitate successful cooperation and coordination [5]. On the one hand, this generates trust and increases coordination and the robustness of the work process against disturbances by means of information exchange and the acquisition of group knowledge $[15,16]$. On the other hand, sharing of the entire knowledge among all team members (generalist team configuration) results in an increased amount of information that needs to be processed by each individual which can lead to information overload [17, 18]. Information overload endangers the effectiveness and efficiency of the team as its members struggle to focus on current tasks when constantly switching between different contexts [5]. A task context defines the setting of task in which needs to be processes. This may some information in order to process a client request or even a change of the physical location to accomplish a task, for instance. However, switching between tasks is causing switching costs.

Contrastingly, specializing on particular areas of competence reduces the cognitive load faced by members of a team [19]. That is, each team member can focus on her specific expertise which reduces the load of information being processed. This distribution of knowledge in specialized teams increases the overall knowledge capacity of the whole team since individual members only have to memorize and process information which is relevant to their areas of expertise [20]. However, specialization potentially makes the team as a system more fragile as it lacks the required redundancy of knowledge to avoid confusion, conflicts, and failures [6].

When switching from a generalist knowledge structure to a specialized one, team member need to sort out irrelevant information on tasks they do not need to process anymore. Hence, intentional forgetting can be defined as this capacity freeing mechanism which allows specializing on certain tasks. In first experimental results it is shown that in dynamic and heavy workload scenarios specialized teams are more efficient than generalist ones in comparison due to task switching costs $[11,21]$. If specialized teams are more 
efficient than generalist ones, then reorganizing in terms of specialization should increase performance.

From an organizational science perspective, specialization is also a common approach for labor division. Thus, there a four benefits connected to a specialization approach [4]: (1) highly specialized positions need only a short period of training; (2) incumbents of specialized position may require only low level qualifications; (3) work seems to be less tiring if only few different tasks have to processed; (4) by working on repeating tasks, workers gain experience and perform more efficient.

However, the challenge of balancing specialization and generalization arises to create robust teams on the one hand and efficient ones on the other hand. In dynamic environments where tasks are suddenly occurring, specialization should increase a team's efficiency. Because if an actor is currently enacting multiple roles at once, then the responsibility for different tasks in dynamic environment may lead to an overload situation. Therefore we analyze intentional forgetting as mechanism of specialization for evaluating different team configurations. We use multiagent-based simulation with a focus on short-term specialization to overcome high workload scenarios. Hence, the next section provides an overview of how organizational models and reorganization approaches are situated in agent research.

\section{Foundations of collaboration}

In organizations, collaboration and distributed task processing allows for a flexible and efficient work environment [4]. From an artificial intelligence perspective, distributed task processing is a key functionality of multiagent systems due to their intelligent and autonomous behavior. In order to cooperatively work together, agents need to coordinate their activities. In MAS research coordination of agents is following to different perspectives: (1) agent-centric or (2) organization-centric [22]. For instance, Wooldridge and Jennings describe a generally applicable process of agent team formation [23]. In that approach teamwork is being initiated if one agent recognize a potential for collaboration because of its own inability of accomplishing a task or due to resource boundaries. Based on this potential, a team is formed by excessive communication and planning in order to accomplish the task at hand. However, this formation process is grounded on collaborative agents which pursue common goals and require aligning task-solving capabilities repeatedly. In large and complex systems this team formation process suffer from its flexibility and neglects goals of the MAS as a society because of individual preferences. Therefore, collaboration and task distribution is a protracted process in agent-centric collaboration.

Hence, organization-centric views on MAS allow for a more consistent view on collaboration. Organizational MAS define a rigid structure to reduce complexity in coordination [24] are defined as "complex entities where a multitude of agents interact, within a structured environment aiming at some global purpose" [22]. In order to structure agents in organization, the commonly used approach are roles [25]. Roles are encapsulating certain behavior which is expected from the agent currently adopting it [26, 27]. In multiagent organizations, the main purpose of an agent is enacting its role(s). Therefore, there are agent models which distinguish between roles and role enactment [28, 29]. That is, agents need to beware of organizational structures and their roles which they are enacting. When agents decide which action to perform next, its behavior need to be aligned with organizational structures and goals. That is, agent need to enact their assigned roles. Once an organizational structure has been designed by role configurations, organizational MAS allow for an economical approach of collaboration in terms of communication costs. However, environments may change over time and be highly dynamic. In such a case, it would be reasonable to adapt an organization. From theory, organizations can be reorganized on two ways [30]:

- Behavioral Change: The organization structure remains but the behavior of the agents is adapted. For instance, this is necessary if a new agent is joining the organization or an agent leaves the organization. Generally, it has be checked if organizational actions are still possible which may require adapting role assignments of the remaining agents. This can be a corrective act to ensure continuous performance. However, behavioral changes have a more temporary character.

- Structural Change: Elements of the organizational structure are modified. That is, organizations are being adapted by adding, deleting or modifying its roles, dependencies, norms, ontologies, or communication primitives. Structural changes ensure a permanent modification of the organization.

Reorganization allows for a goal-oriented response to changing environmental influences so that the organization's long-term aims can be achieved. 
Reorganization can either be controlled by a central agent or decentralized by group of agent which requires communication and coordination [30]. When reorganization becomes necessary, both approaches include searching for an optimal structure [31, 28]. However, an optimal search for role assignments and a organizational structure is costly in terms of time and resources. Therefore they are less suitable for dynamic environments (such as emergency response scenario) to allow for quick adaptations to increase performance.

In order to allow for an intentional forgetting approach in an multiagent-based simulation model, we focus on behavior-based reorganization mechanisms for adaptation at runtime. Adapting the behavior of an agent in an organizational MAS is concerned with its reasoning capabilities, i. e., the process of action selection [32]. Action selection in agent organization is controlled by roles the agent is currently enacting [28]. Therefore we present a concept for an agent-based model of how role assignment can be adapted to allow for short-term specialization by means of intentionally forget irrelevant information.

\section{Role assignment adaptation for intentional forgetting}

In this section we present intentional forgetting as a mechanism for specialization in an intelligent agent model. In order to specialize agents, role assignments are adapted to induce intentional forgetting. Section 4.1 describes an Belief-Desire-Intention agent architecture which allows for a combination of organizational structures and agent technology. Based on this model, section 4.2 describes an intentional forgetting role assignment adaptation mechanism for this agent model.

\subsection{Organizational BDI agent}

For modeling organizational behavior, a Belief-Desire-Intention (BDI) agent architecture is used. BDI agents model human-like decision making processes by a goal oriented approach [33]. This architecture provides mental states for the agents knowledge (beliefs), its goals (desires), and its intentions. Due to their universal applicability, BDI agents are used in different domains. By combining deduction and means-end-reasoning, they can accomplish complex tasks and reason about extensive environments. BDI agents have been successfully applied to social simulation [34]. In order to utilize the BDI-paradigma, the following paragraphs introduce the intentional forgetting agent (IFA).

The main component of IFA is its local state $L$ which represents necessary knowledge about the environment and its own capabilities such as plans. Based on $L$ the agent derive decision such as actions selection or communication. A definition of local states of an IFA is given by a 5-tuple $L=\langle B, D, I$, Plan, $\gamma\rangle$ (based on [35]). It contains set for beliefs (B), desires (D), intentions (I), plans (Plan), and relevance function of desire $(\gamma)$.

When being in an organization, IFA adopt and enacts roles. As pointed out in the previous section, roles define a certain behavior which other members of an organization expect from this role. In this model, roles are defined by Role $=\left\langle C_{\left.C a p s_{\text {Role }}, I D\right\rangle}\right.$ with $C_{\text {Caps }}$ Role being a set of required capabilities and $I D$ being a unique identifier for distinguishing purposes. A capability for Agent $i$ is defined as: $\operatorname{Cap} i \varphi \leftrightarrow$ $\varphi \in \operatorname{Plan}^{i}$. That is, if an agent $i$ has the capability of achieving $\varphi$ then it has a plan which allows it to achieve $\varphi$. The set of capabilities of an agent $i$ is denotes by $\mathrm{Caps}_{i}$. In order to behave according to a role definition, i.e., enacting as other would expect, an agent need to percept environment aspects for deriving its actions.

Role enactment $R E$ implements a role assignment for a specific role $R$ to an agent $i: R E=\langle R, i|$ $\left.\operatorname{Caps}_{R} \subseteq \operatorname{Caps}_{i}\right\rangle$. RE ensures that only agents with the required capabilities can enact specific roles they are assigned to. Furthermore it allows for registering which agent currently enacting which roles by a set of all $R E$ configuration within an agent organization.

Figure 1 presents the reasoning process of an organizational IFA. An IFA percepts its environment via a "Perception"-component. This component transforms external information into an internal representation which can be interpreted by the agent's reasoner. This perception is then transformed into the agent's belief base. For this purpose a Belief-Revision-Function ( $b r f$ ) is utilized. Based on the perception, a brf allows an IFA to keep track of environmental changes. When enacting a certain role, an IFA needs to reason about tasks at hand. In order to allow for an adaptive agent architecture, the $b r f$ of an IFA is divided into role specific functions. This is necessary for an agent to focus on relevant perception (cf. Section 4.2). Based on the new beliefs, an agent must reconsider its current intentions which might not be adequate given the current situation. Afterwards, new options for the current situation are being generated and written to a goal list. By filtering options, an agent determines its new set of intentions. An intention is represented by a combination of a desire and a plan. If the agent has decided on committing a specific intention, it executes its the attached plan. The selected intention contributes to the desire with the highest priority. The next Section describes how this agent model is used to model 


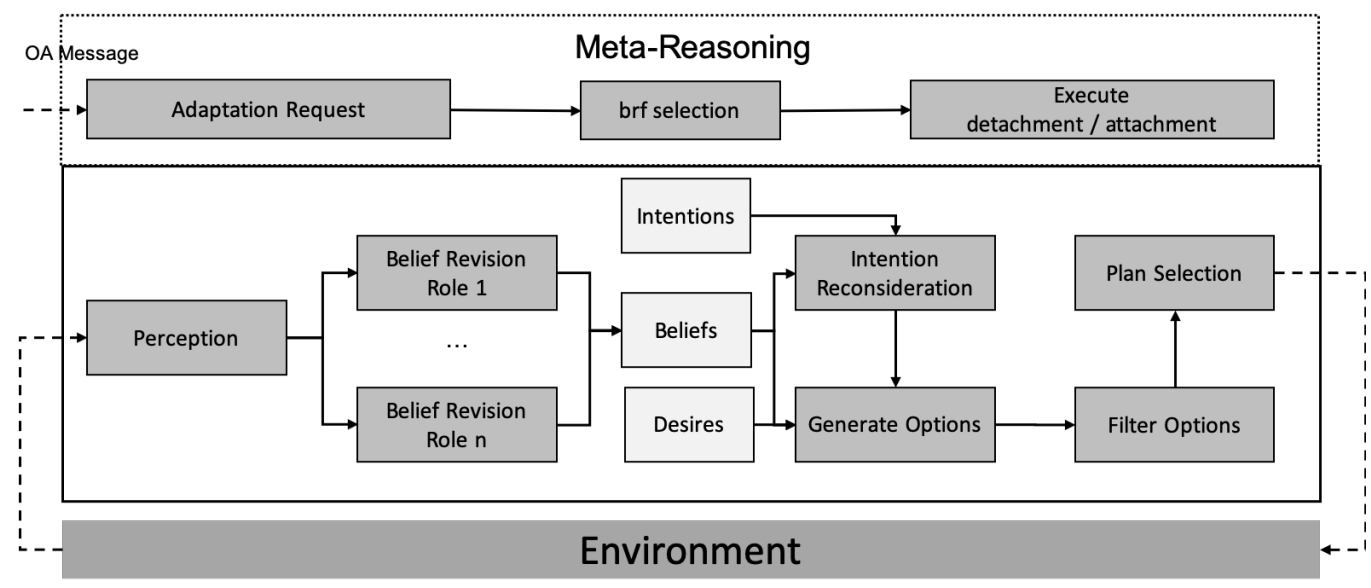

Figure 1. Role enactment in BDI reasoning

intentional forgetting and role adaptation by utilizing distinct belief-revision-functions.

\subsection{Adaptation of role assignment}

As presented in Section 2, intentional forgetting in teamwork is defined by specializing team members. That is, team member only need to focus on their tasks which they are responsible for. In an organizational structure, responsibilities and expectation are predefined by roles. Hence, when modeling intentional forgetting for organizations, it is necessary to adapt the assignment of roles for the team members. If an agent enacts less roles, they are more specialized on specific tasks.

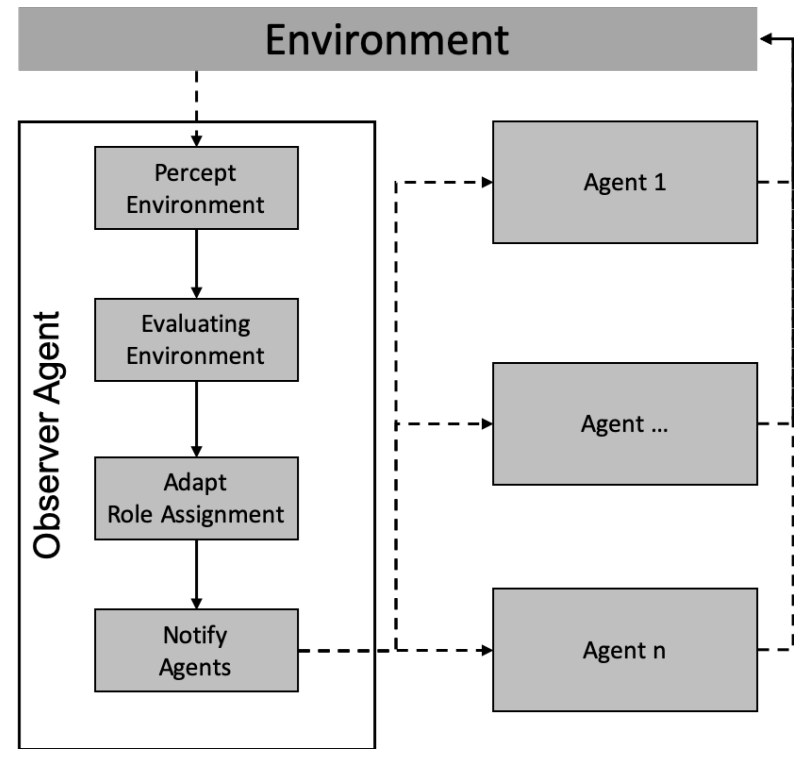

Figure 2. Adapting role assignment by an observer agent
In this model, role assignment adaptation is triggered by a centralized instance, namely the observer agent (OA). The model of the OA is depicted in Figure 2. This agent observes the environment constantly. By perceiving the environment, the OA evaluates each environment state $E$. The evaluation is based on performance indicators (PI). A PI is defined PI : $E \rightarrow \mathbb{R}$. For each specific environment numerous PI can be defined. For instance, an indicator may evaluate team performance whereas a different one measure individual performance. However, PIs are related to the identification of high workload situations. If one or more $P I$ exceeds a certain threshold, a role enactment adaptation is triggered. In this model, reorganizing relies on the assumption of specializing teams to gain performance. That is, if a PI exceeds a threshold, the role assignment is adapted. For adapting role assignment, certain strategies can be applied. For instance, a complete generalized team can be gradually adapted until each agent only enacts a single role (complete specialization). To which point a specialization is necessary has to be evaluated within each application. Figure 3 shows as a reorganization of roles. In this example, a team of three agent with a generalist role enactment configuration (left side) is reorganized by specializing on two of three possible roles (right side).

In order to configure role assignment adaptation, the degree of specialization within a team is calculated (cf. equation 1). It has to be stated that agents need to enact at least one role in order to be part of the organization as well as a role need to enacted by at least one agent.

$$
\text { Specialization Degree }=1-\frac{\# \text { Roles }_{\text {Agent }}}{\# \text { Roles }_{\text {Total }}}
$$



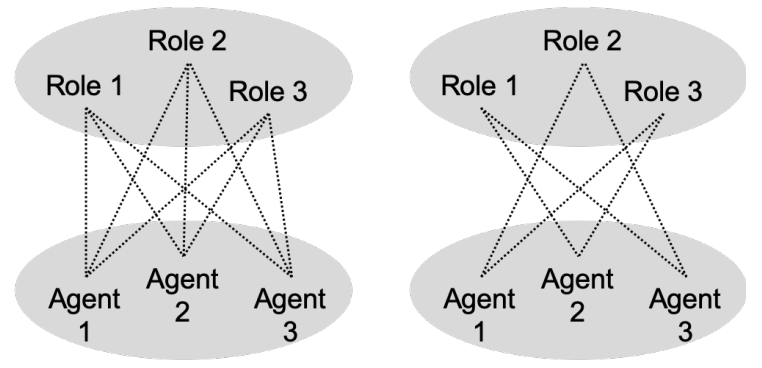

Figure 3. Adapting role assignment example

The notification of each agent to change their internal reasoning is communicated by message passing. These messages from the $\mathrm{OA}$ are processed by a meta-reasoner (MR) of each agent. Each adaptation message contain information about what roles the agent is no longer enacting or which role is going to added to an agents repertoire. A MR is isolated from an agents deliberation process. In order to adapt to the new role configuration of the team, the MR adapts the brf connections of an agent. Regarding the example in Figure 3, the MR of "Agent 1" would disconnect the brf for "Role1". That is, "Agent 1" is no longer assigned to "Role 1" and does not focus on any information regarding the tasks connected to this role. Hence, this agent's knowledge-base is reduced by restricting the roles it is enacting, the agent intentionally forgets.

\section{Case-Study: Adaptation performance of agent teams}

This Section provides a case-study to evaluate the intentional-forgetting-based reorganization model. For testing the model we utilize a serous game environment [36] in an emergency response scenario. In general, emergency scenarios are a common application for intelligent agents [37]. In these, time is a critical factor and therefore decision have to be made quickly. Hence, such an scenario is interesting for balancing generalization and specialization. On the one hand, operators need clear responsibilities to act quickly and to avoid failure due to a large communication overhead. That is, they would benefit from a specialized labor division. On the other hand, robustness of the team is a must to allow for action when a part of the team becomes unavailable or tasks are unequally distributed. Since redundancy of actors is costly, redundancy in overlapping capabilities is more economic.

In particular, this very scenario has been successfully applied for agent-based as well as psychological teamwork experiments to test the effects of different role configurations in teams. Timm et al., show that specialists are making less mistakes in this emergency response scenario than generalists [11]. Therefore runtime adaptation of role assignment based on intentional forgetting is a promising approach.

The environment configuration for testing the adaptability of the agent-based simulation model is describes in Section 5.1. In Section 5.2 results for simulating different team configurations as well as adaptation strategies are presented and discussed.

\subsection{Serious game environment}

In this serious game, agents are situated in a grid-based environment in which suddenly occurring fires need to be extinguished. Agents can extinguish fires by using firetrucks and positioning them on the fire location. Each Firetruck has a predefined amount of extinguishing capacity, i.e., it can extinguish a certain amount of fire before it needs to be refilled. Refilling firetrucks can be done by selecting a particular watering place located at the center of the environment. The initial scenario is depicted in Figure 4.

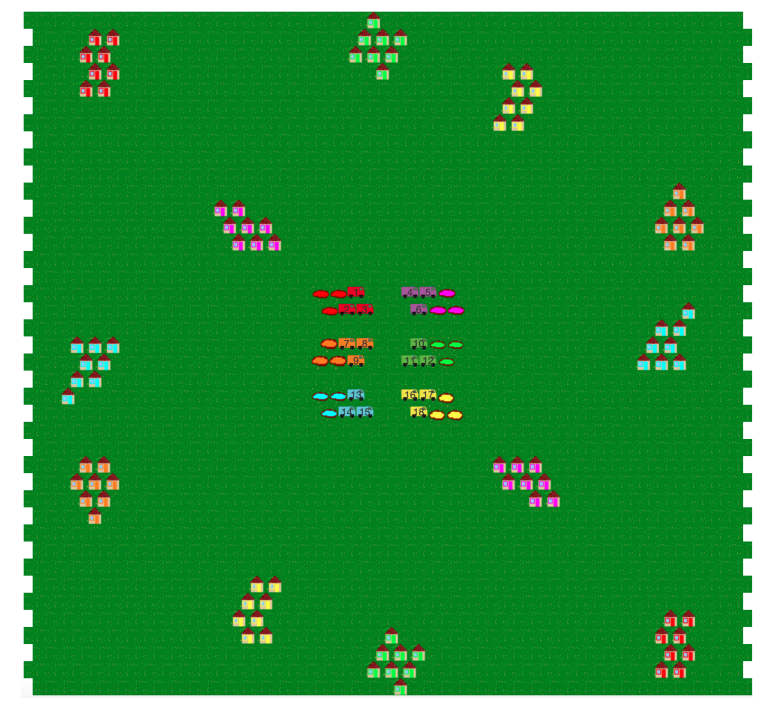

Figure 4. Emergency response scenario configuration

A role is defined as a set of extinguishing capability. For each housing group a different capability is needed. In this simulation, each agent has technically the capability to extinguish all fire types. If a fire starts a specific group of houses, then only agents enacting the necessary role are allowed to extinguish the fire. That is, if a fire starts which an agent is not specialized on, the brf for this role is not attached. In theory, specialization is causing efficiency benefits because there are a few or sometimes no task switching costs [5, 4]. In this scenario, task switching costs are occurring 
when switching between fire locations. Agents need to prepare for the new fire and drive to the new location.

In order to reconfigure role assignment, there is a central role managing agent which is constantly observing the environment. At the beginning of each simulation run, all players are configured as generalists, i.e., every player is responsible for all occurring fires. This guarantees high availability of firetrucks and a robust, i.e., redundant team configuration. However, when dealing with a high workload situation, the managing agent specializes team members on certain areas if observing performance losses. In this scenario, performance is measured either for effectivity as well as efficiency. For effectivity, performance is defined as Effectivity PI $=\frac{\# \text { extinghuished Fires }}{\text { \#total Fires }}$. This indicator is representing team performance between $[0 ; 1]$. A score of 1 indicates a perfect performance because all fires are extinguished in the given time. The efficiency, is measured by summarizing all distances for all vehicles. The effectivity performance indicator is used to trigger reorganization events. Efficiency is only a secondary indicator which directly results from focusing on housing groups nearby. In order to configure different role combinations, housing groups are clustered based on their location. Hence, groups with a low euclidean distance are coupled. This ensures that agents focus on roles with low task switching costs.

\subsection{Simulation experiments and results}

This section provides an overview on experiments as well as their results for reorganizing role assignment in teams by initiating intentional forgetting processes. Based on first results in prior studies, we investigate reorganization in the terms of specializing on certain areas of expertise. As these studies showed, specializing is more efficient regarding error values [11, 21]. For testing the adaptation approach we define two hypotheses:

1. In complex work environments specialist role assignment configurations will perform better than generalist knowledge structure according to performance indicator.

2. Based on the first hypothesis, gradual adaptation by increasing the degree of specializing will also have positive and significant effects of the team's performance.

In order to analyze the hypotheses a sensitivity analysis of the simulation model is conducted. The fire fighting serious game is configured with three playing agents which control three fire trucks each. In total there are 12 housing groups, i. e., roles an agent is allowed to enact. This configuration is derived from previous studies and allows for varying workload scenarios [11]. The results are generated by repeating each simulation run 500 times.

Figure 5 shows the results of the conducted simulation experiments. In the bottom right corner, the results for comparing generalist, specialist as well as the gradual reorganization approach is shown. Based on the effectivity measurement for this scenario (burned houses), specialist perform best and generalists worst. The gradual reorganization is in between. Besides reorganizing teams to a full specialist, teams are adapted gradually by its degree of specialization (cf. Equation 1).

The results show the average performance measurement within simulation runs over time. This not only supports the main hypothesis that specialist perform significantly better $(p=0,12 \%)$, but also shows potential for reorganizing team configurations at runtime. The deviation of the different team configuration is shown in Figure 5 in the top left corner. It shows a standard deviation of: $\sigma_{\text {Generalists }}: 0.075, \sigma_{\text {Specialists }}: 0.11$, and $\sigma_{\text {Reorganization }}: 0.077$ with specialist configuration being the most deviating configuration. This is caused by the dynamic environment in which tasks may not be equally distributed across the team.

Besides performance indicator for effectivity, efficiency of the reorganization was also measured (cf. Figure 5 top right corner). These results show that specialist team configurations drive significantly less across the environment. The efficiency is measured using cumulative distances of each team. That is, each distance is measured in grid points a team has drove its trucks in a $40 \times 40$ environment as depicted in Figure 4 . The deviation across each team configuration is: $\sigma_{\text {Generalists }}: 21.9, \sigma_{\text {Specialists }}: 27,1$, and $\sigma_{\text {Reorganization }}: 21,7$. Although the differences in effectivity being not as distinct, reorganizing teams have a big impact on the efficiency. Especially specialist team configuration use resources more economical. Especially in scenarios where resource consumption is restricted or expensive such as in manufacturing processes or in logistics efficiency is a key principle.

However, teams perform differently in more or less dynamic environments. Hence, the reorganization approach is tested against generalization in environments with varying dynamics. In this fire fighting serious game, dynamics can be modeled by fire location as well as firing time of the houses. If more fire with a shorter burning time occur, then the environment is getting more dynamic for the players. 


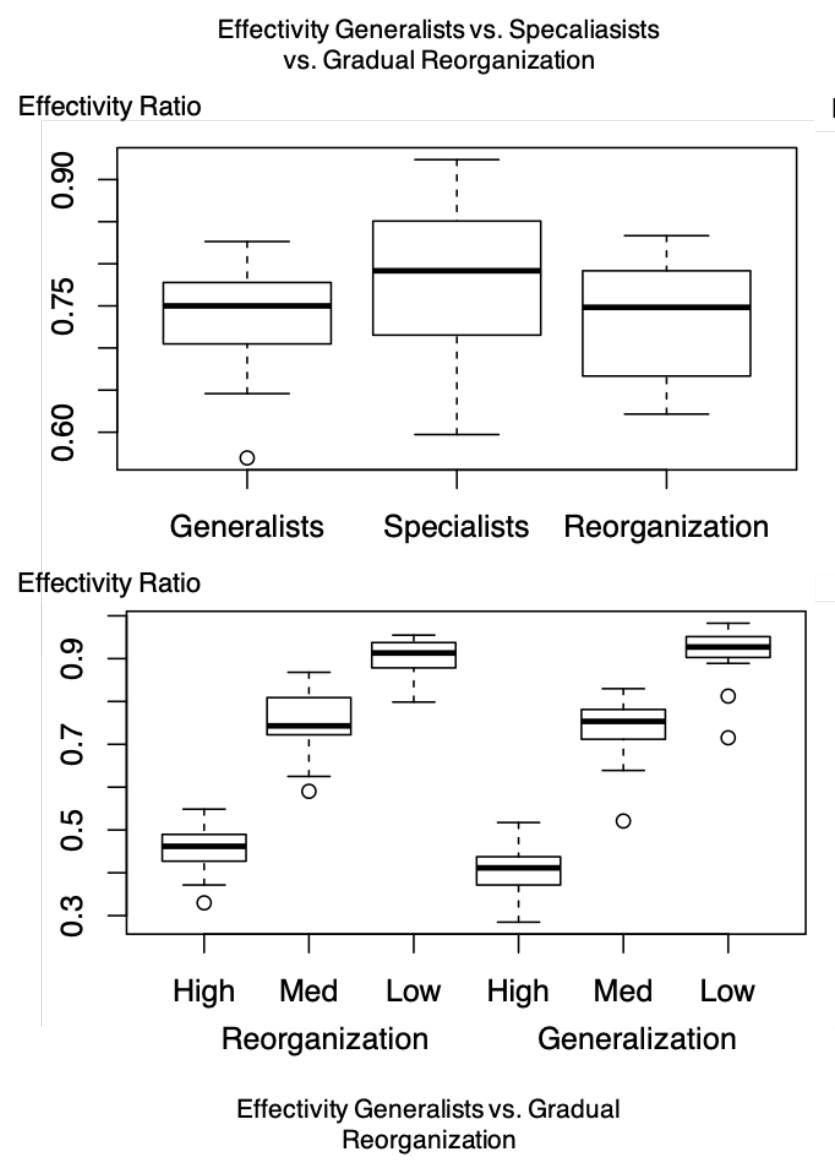

Efficiency Generalists vs. Specaliasists

vs. Gradual Reorganization

Distance in Grids

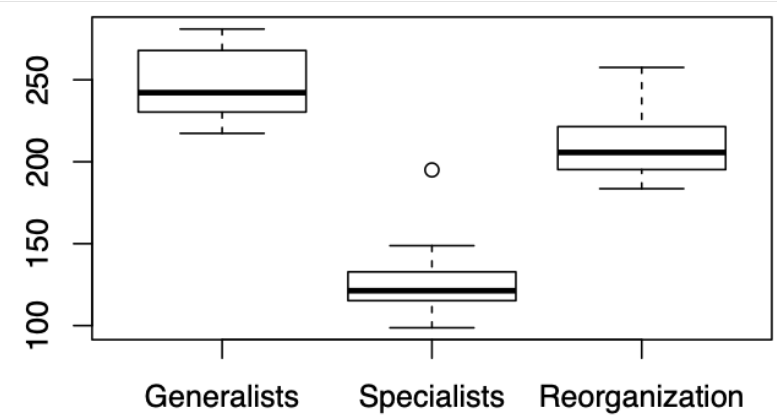

Effectivity Ratio

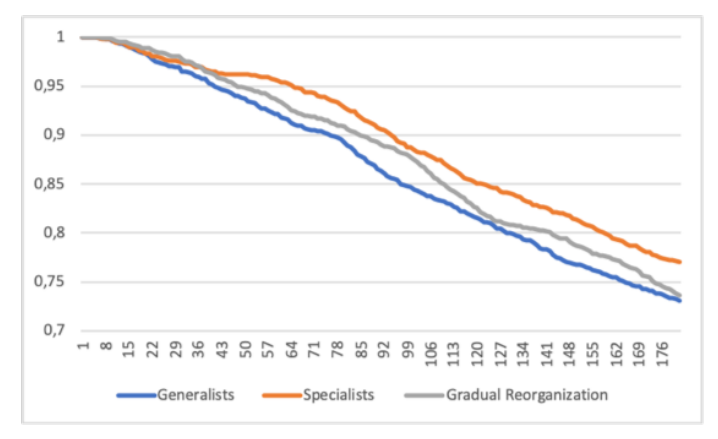

Effectivity Generalists vs. Specaliasists vs. Gradual Reorganization in Simulation run

Figure 5. Experiment results for analyzing role assignment adaption

For the simulation, dynamic is varied in the classes of low, medium, and high. While a high dynamic being twice as much fires and a halved burn down time of houses as in medium dynamic preferences (respectively for medium and low dynamic). The standard deviation within the different teams: (1) Reorganization $\sigma_{H i g h}: 0.051, \sigma_{\text {Medium }}: 0.069$, and $\sigma_{\text {Low }}: 0.037$ as well as (2) Generalists $\sigma_{\text {High }}: 0.056$, $\sigma_{\text {Medium }}: 0.063$, and $\sigma_{\text {Low }}: 0.051$, In high dynamic environments, the reorganization approach shows higher effectivity than the generalist team configuration (significant $p$-value $=0.00171$ ). However, in low dynamic environments both approaches achieve almost identical results. This supports the assumption that context switches have a significant impact on effectivity in organizational MAS.

In total, intentional forgetting triggered by role assignment adaptation is a promising approach for evaluating labor division concepts within specialist and generalist team configuration. By gradually focusing on specific areas of interest, the performance of the team can be increased significantly. Especially in dynamic environments, the reorganization approach shows its potential for team efficiency and effectivity. This simulation is a first step towards the vision of modeling and evaluating team adaptation. Since the context switching cost and coordination effort are determined by the simulation environment, this agent model can be calibrated towards a more realistic teamwork scenario.

\section{Conclusions and future work}

Modern organizations especially in socio-digital environment as in an Industry 4.0 context are dealing with a complex and dynamic environment. In these scenarios, digital representatives are used to complement machines as well as humans in an organizational structure. In dynamic situations, tasks need to be distributed across the team. For distributing tasks successfully, organizations provide a structure based on roles to control responsibilities and expectations from its incumbents. However, if actors enact multiple roles, their responsibilities for task-processing is rising. Especially when 
tasks are being generated more frequently. From an organizational perspective, specializing on particular roles allows for more efficiency due to less switching between different tasks. This coincides with psychological research in which focus allows for an increased cognitive capacity. Hence, we define intentional forgetting as a deliberate specialization on roles. If actors only need to remember and store information about a smaller set of roles they are enacting, then reduced task switching costs lead to a higher performance of the team as a whole.

Therefore we utilize intentional forgetting as a mechanism to adapt role enactment configuration for agent teams to cope with high workload scenarios. If agent focus on certain task which is induced by specializing on single roles, task switching costs are reduced. For adapting role assignments in agents, the intentional forgetting agent (IFA) is developed. The IFA is modularized by a belief-revision-function for each role. This allows for a goal directed focus on roles, respectively tasks, the agent needs to accomplish. By detaching such belief-revision-functions the agent specializes on roles. In order to adapt an agent's reasoning process, a meta-reasoning component is complementing the agent. This component can detach or attach belief-revision-functions. Such behavioral reorganization is triggered by an observer agent which manages the role enactment of each agent by measuring its performance. The reorganization of agent teams by adapting role assignment is tested using a case-study based on a dynamic emergency response scenario in which agents need to fight fires. A role is defined as a set of fire extinguishing capabilities. Since the fires are distributed across the environment, agents have driving costs when switching between them. The case-study starts with a generalist role assignment configuration which is specialized at runtime. The performance of the team is measured by effectivity (burned houses) as well as efficiency (cumulative driving routes). The results for different simulation runs show a significant performance benefit of specialized teams over generalist ones (effectivity as well as efficiency). Moreover, a continuous reorganization strategy is also more effective than generalist configurations.

By specializing agents the team gets more fragile and is prone to disturbances, e. g., an agent leaves the organization. Furthermore, specialization leads to unilateral task-processing. If agents execute plans repeatedly they are gaining experience knowledge. But the acquisition of such experience knowledge is limited when only doing specialized tasks. Hence, we want to extend our approach by integrating experience of task-related knowledge. In order to validate the results for human actors, we plan on conducting laboratory experiments in a socio-digital environment.

\section{Acknowledgments}

The project AdaptPRO: Adaptive Process and Role design in Organizations (TI 548/-1) is funded by the German Research Foundation (DFG) within the Priority Program "Intentional Forgetting in Organizations" (SPP 1921).

\section{References}

[1] F.-Y. Wang, "The emergence of intelligent enterprises: From cps to cpss," IEEE Intelligent Systems, vol. 25, no. 4, pp. 85-88, 2010.

[2] Z. Liu, D.-s. Yang, D. Wen, W.-m. Zhang, and W. Mao, "Cyber-physical-social systems for command and control," IEEE Intelligent Systems, vol. 26, no. 4, pp. 92-96, 2011.

[3] E. H. Durfee, V. R. Lesser, and D. D. Corkill, "Trends in cooperative distributed problem solving," IEEE Transactions on knowledge and data Engineering, vol. 1, no. 1, pp. 63-83, 1989.

[4] A. Kieser and P. Walgenbach, Organisation. Schffer-Poeschel Verlag, 2010.

[5] T. Ellwart and C. H. Antoni, "Shared and distributed team cognition and information overload. evidence and approaches for team adaptation.," in Information and Communication Overload in the Digital Age, IGI Global, 2017 in press.

[6] D. M. Wegner, "Transactive memory: a contemporary analysis of the group mind," in Theories of group behavior (B. Mullen and G. R. Goethals, eds.), pp. 185-208, New York: Springer, 1987.

[7] J. M. Golding and C. M. MacLeod, "There's more to intentional forgetting than directed forgetting: An integrative review," in Intentional Forgetting, pp. 71-114, Psychology Press, 1998.

[8] T. Ellwart, A.-S. Ulfert, C. H. Antoni, J. Becker, C. Frings, K. Göbel, G. Hertel, A. Kluge, S. M. Meeßen, C. Niessen, et al., "Intentional forgetting in socio-digital work systems," AIS Transactions on Enterprise Systems, vol. 4, no. 1, 2019.

[9] I. J. Timm, S. Staab, M. Siebers, C. Schon, U. Schmid, K. Sauerwald, L. Reuter, M. Ragni, C. Niederée, H. Maus, G. Kern-Isberner, C. Jilek, P. Friemann, T. Eiter, A. Dengel, H. Dames, T. Bock, J. O. Berndt, and $\mathrm{C}$. Beierle, "Intentional forgetting in artificial intelligence systems: Perspectives and challenges," in $K I$ 2018: Advances in Artificial Intelligence (F. Trollmann and A.-Y. Turhan, eds.), (Cham), pp. 357-365, Springer International Publishing, 2018.

[10] I. J. Timm and L. Reuter, "The role of knowledge in intelligent agents," in Proceedings of the 7th Workshop on Dynamics of Knowledge and Belief (DKB-2018) and the 6th Workshop KI \& Kognition (KIK-2018) co-located with 41st German Conference on Artificial Intelligence (KI 2018), Berlin, Germany, September 25, 2018., pp. 55-67, 2018.

[11] I. J. Timm, L. Reuter, J. O. Berndt, A. Ulfert, T. Ellwart, and C. H. Antoni, "Analyzing the 
effects of role configuration in logistics processes using multiagent-based simulation: An interdisciplinary approach," in 52nd Hawaii International Conference on System Sciences, HICSS 2019, Grand Wailea, Maui, Hawaii, USA, January 8-11, 2019, pp. 1-10, 2019.

[12] V. B. Hinsz, R. S. Tindale, and D. A. Vollrath, "The emerging conceptualization of groups as information processors," Psychological bulletin, vol. 121, no. 1, pp. 43-64, 1997

[13] S. W. J. Kozlowski and D. R. Ilgen, "Enhancing the effectiveness of work groups and teams," Psychological Science in the Public Interest, vol. 7, no. 3, pp. 77-124, 2006.

[14] T. Ellwart, U. Konradt, and O. Rack, "Team mental models of expertise location: Validation of a field survey measure," Small Group Research, vol. 45, no. 2, pp. 119-153, 2014.

[15] A. Wiedow, U. Konradt, T. Ellwart, and C. Steenfatt, "Direct and indirect effects of team learning on team outcomes: A multiple mediator analysis," Group Dynamics: Theory, Research, and Practice, vol. 17, no. 4, pp. 232-251, 2013.

[16] R. Oertel and C. H. Antoni, "Phase-specific relationships between team learning processes and transactive memory development," European Journal of Work and Organizational Psychology, vol. 24, no. 5, pp. 726-741, 2015.

[17] T. Ellwart, C. Happ, A. Gurtner, and O. Rack, "Managing information overload in virtual teams: Effects of a structured online team adaptation on cognition and performance," European Journal of Work and Organizational Psychology, vol. 24, no. 5, pp. 812-826, 2015.

[18] M. J. Eppler and J. Mengis, "The concept of information overload: A review of literature from organization science, accounting, marketing, mis, and related disciplines," The Information Society, vol. 20, pp. 325-344, 2004.

[19] A. B. Hollingshead, "Communication, learning, and retrieval in transactive memory systems," Journal of Experimental Social Psychology, vol. 34, pp. 423-442, 1998.

[20] A. B. Hollingshead, N. Gupta, K. Yoon, and D. P. Brandon, "Transactive memory theory and teams: Past, present and future," in Theories of team cognition: Cross-disciplinary perspectives (E. Salas, S. M. Fiore, and M. P. Letsky, eds.), pp. 421-455, New York: Routledge, 2012.

[21] L. Reuter, J. O. Berndt, and I. J. Timm, "Towards simulation-based role optimization in organizations," in KI 2017: Advances in Artificial Intelligence - 40th Annual German Conference on AI, Dortmund, Germany, September 25-29, 2017, Proceedings, pp. 359-365, 2017.

[22] V. Dignum, "The role of organization in agent systems," in Handbook of Research on Multi-Agent Systems: Semantics and Dynamics of Organizational Models, pp. 1-16, IGI Global, 2009.

[23] M. Wooldridge and N. R. Jennings, "Formalizing the cooperative problem solving process," Synthese Library, pp. 143-162, 1997.

[24] M. Schillo, B. Fley, M. Florian, F. Hillebrandt, and D. Hinck, "Self-organization in multiagent systems: from agent interaction to agent organization," in Third International Workshop on Modelling Artificial Societies and Hybrid Organisations (MASHO), pp. 37-46, 2002.
[25] M. Hannoun, J. S. Sichman, O. Boissier, and C. Sayettat, "Dependence relations between roles in a multi-agent system," in Multi-Agent Systems and Agent-Based Simulation (J. S. Sichman, R. Conte, and N. Gilbert, eds.), (Berlin, Heidelberg), pp. 169-182, Springer Berlin Heidelberg, 1998.

[26] J. Ferber and G. Weiss, Multi-agent systems: an introduction to distributed artificial intelligence, vol. 1. Addison-Wesley Reading, 1999.

[27] L. R. Coutinho, J. S. Sichman, and O. Boissier, "Modelling dimensions for agent organizations," in Handbook of research on multi-agent systems: Semantics and dynamics of organizational models, pp. 18-50, IGI Global, 2009.

[28] V. Dignum and F. Dignum, "A logic of agent organizations," CoRR, vol. abs/1804.10817, 2018.

[29] J. J. Odell, H. V. D. Parunak, and M. Fleischer, "The role of roles in designing effective agent organizations," in International Workshop on Software Engineering for Large-Scale Multi-agent Systems, pp. 27-38, Springer, 2002.

[30] M. Dignum, E. Sonenberg, and F. Dignum, "Dynamic reorganization of agent societies," in Proceedings of workshop on coordination in emergent agent societies, 2004.

[31] B. Dunin-Keplicz and R. Verbrugge, Teamwork in multi-agent systems: A formal approach, vol. 21. John Wiley \& Sons, 2011.

[32] M. Wooldridge, Reasoning about Rational Agents. Cambridge: The MIT Press, 2000.

[33] S. Rao and M. P. Georgeff, "Bdi agents: From theory to practice," in Proceedings of the First International Conference on MultiAgent Systems (ICMAS 1995) (V. R. Lesser and L. Gasser, eds.), (Boston), pp. 312-319, The MIT Press, 1995.

[34] C. Adam and B. Gaudou, "Bdi agents in social simulations: a survey," The Knowledge Engineering Review, vol. 31, no. 3, pp. 207-238, 2016.

[35] I. J. Timm, Dynamisches Konfliktmanagement als Verhaltenssteuerung Intelligenter Agenten. Dissertation in der Knstlichen Intelligenz (DISKI) Aka Verlagsgruppe, 2004.

[36] L. Reuter, J. O. Berndt, and I. J. Timm, "Challenges of simulating teamwork in organizational scenarios," in 2017 Winter Simulation Conference, WSC 2017, Las Vegas, NV, USA, December 3-6, 2017, pp. 4542-4543, 2017.

[37] R. Buettner and H. Baumgartl, "A highly effective deep learning based escape route recognition module for autonomous robots in crisis and emergency situations," in 52nd Hawaii International Conference on System Sciences, HICSS 2019, Grand Wailea, Maui, Hawaii, USA, January 8-11, 2019, pp. 1-8, 2019. 